\title{
When individuals senesce: the 'Florida effect' on stable populations of territorial, long-lived birds
}

\author{
Vincenzo Penteriani, Miguel Ferrer, Fermín Otalora and María del Mar Delgado
}

V. Penteriani (penteriani@ebd.csic.es) and M. del Mar Delgado, Dept of Conservation Biology, Estación Biológica de Donana, C.S.I.C., Pabellón del Perú, Avda. María Luisa s/n, ES-41013 Seville, Spain. - M. Ferrer, Dept of Biodiversity Conservation, Estación Biológica de Doñana, C.S.I.C., Avda. María Luisa s/n, Pabellón del Perú, ES-41013 Seville, Spain. - F. Otalora, Laboratorio de Estudios Cristalográficos, IACT (CSIC/Univ. of Granada), Campus Fuentenueva, Facultad de Ciencias, ES-18002 Granada, Spain.

\begin{abstract}
Senescence can be defined as the entire set of age-related changes that affect both vitality and function, one of which is within-individual age-related decline in reproduction. This factor is crucial for population persistence, because the senescence of individuals of a population can increase the likelihood of local extinction. Using simulations based on longterm information on a small metapopulation of a long-lived bird species, we highlight two mechanisms able to engender senescence in both breeders and floaters (i.e. non-breeding individuals) of the same population. We define 'floater shortage senescence' as breeder senescence due to low juvenile replacement rates because of high floater mortality during dispersal. Less obviously, senescence can also occur with very low floater mortality rates, and when breeding populations are remarkably free from factors that could cause catastrophic population decimation. In this scenario, low mortality in reproductive areas results in territory owners in breeding populations being characterised by progressively older breeding individuals, and old individuals waiting for a breeding opportunity: a phenomenon we refer to as the 'Florida effect'. Consistent with current views that adaptive death plays a crucial role in population dynamics, it seems reasonable to suppose that, under stable environmental conditions, the evolution of some mating mechanisms could limit the senescence of breeding individuals in a population, allowing the pool of breeding individuals to be refreshed by selection of younger breeders.
\end{abstract}

A peculiar life history characteristic of iteroparous organisms is that their breeding performance varies with age, often being high in early life and low in later life (CluttonBrock 1988, Newton 1989, Sæther 1990). Significant within-individual age-related declines in reproduction are probably due to degenerative senescence (sensu Abrams 1991), which is an age-related decline in physiological and cellular function that occurs in many vertebrate taxa (Nussey et al. 2006). More generally, individual senescence can be defined as the entire set of age-related changes that affect both vitality and function (Monaghan et al. 2008), with one of the most important being an increase in mortality rate as a function of time (Partridge and Barton 1993, Nisbet 2001, but see Hamilton 1966, Hayflick 2000, Vaupel et al. 2004).

Since the publication of the seminal works of Hamilton (1966), Curio (1983) and Clutton-Brock (1984), considerable research has been directed at understanding the mechanisms underlying high reproductive success in young age groups, and the factors leading to declines in offspring production later in life (Angelier et al. 2006, Hatch and Westneat 2007), as well as the relationships between investment in reproduction and lifespan (Ricklefs and Cadena 2007). The influence of age on survival and reproductive performance is crucial in animal life histories because it affects reproductive strategies (Roff 1992, Stearns 1992, Catry et al. 2006), and consequently alters population dynamics. Despite the large body of research into individual-level mechanisms and factors affecting agerelated reproductive success, more information is needed on the mechanisms and factors underlying the occurrence and effects of individual senescence at the population level (but see Le Page and Cury 1995, Ronce et al. 1998, Doncaster 2003, Williams and Day 2003, Dytham and Travis 2006). Moreover, the existing theoretical literature is limited to linkages of senescence with kin (Bourke 2007) and sexual selection (Bonduriansky et al. 2008). As recently underlined by Monaghan et al. (2008, p. 375), "more interaction with theories of the evolution of senescence and other areas of evolutionary theory is also needed'.

For example, because the individuals in a population tend to reproduce less well at older life stages (i.e. when they senesce), what processes and/or factors may determine the prevalence of old breeders? As many animal populations have demographic characteristics that differ with age, it may be critical to consider age structure and effects when analyzing population dynamics and patterns. This aspect is crucial for population persistence, because individual 
senescence can increase the likelihood of population extinction: small reductions in breeder survival and fecundity represent serious threats for small (meta)populations (Simons 1984).

We used object-oriented simulations to explore the possibility that in territorial long-lived bird species the interactions among some life-history traits of breeders and floaters (i.e. non-breeding individuals) within small populations may produce scenarios in which individuals senesce in the population. On the basis of long-term data we provide evidence for two potential mechanisms of senescence in individuals of the population. Territoriality and life expectancy of individuals are crucial elements of our simulation because, due to strong territoriality and individual lifespan, non-breeders may remain floating for long periods before becoming breeders in the reproductive pool. On the one hand, the queuing time for breeding may be long (i.e. new breeders are old floaters); on the other, high rates of non-breeder mortality may be associated with long periods of floating. Although the scenario we simulated is mainly typical of (large) bird species with the life-history characteristics noted above, we hypothesize that it also explains individual senescence for those long-lived vertebrate species in which territoriality determines the appearance of a pool of floaters waiting for breeding opportunities.

\section{The modelling approach: starting from a strong empirical background}

Our individual-based model was built using information from a database comprising 100 years of information on a metapopulation of the Spanish imperial eagle, Aquila adalberti, in Doñana (southwestern Spain). This population has been studied since 1890, and is a unique long-term data set that provides a valuable tool for investigations of the dynamics and trajectories of small populations. For the current purpose we used the most complete information from the available data, i.e. from 1959 until 2004. The data used in this study (Ferrer and Penteriani 2008) were: (a) obtained from the Doñana Biological Station archives (1959-1985); and (b) collected in the field by one of the authors (MF, 1985-2004). The whole area was surveyed at the beginning of each breeding season (from territorial demarcation and nest selection to the courtship period) to determine whether pairs were present in their breeding territories. The sedentary behaviour of this species greatly facilitates the observation of breeding pairs. Therefore, it is likely that all breeding attempts were detected, as were pairs that did not breed. When nestling age enabled approach without risk to the birds, nests were visited regularly to check the progress of reproduction and record the number of fledglings. We considered a breeding attempt to have been successful when the nestlings reached the age of 50 days. The calculation of productivity did not include data on pairs that received less than two visits during the reproductive period; years lacking data for the beginning of the breeding season were also excluded. With a current worldwide population of less than 150 pairs, this eagle is the most endangered bird of prey in Europe and one of the most threatened raptors in the world (Ferrer 2001). The species is characterised by a low reproductive rate (an average of 0.75 chicks per pair per year), an immature phase of 3-5 years (most frequently five in saturated populations), and a maximum longevity of 22 years. We report here only the information from our simulation algorithms and parameters directly related to the topic of the present work (the complete models having been described in detail in Penteriani et al. 2005a, 2005b, 2006, 2008). As there is no precise information on the real rate of age-specific reproductive decline for $\mathrm{A}$. adalberti, this parameter was not included in the model. However, the main aim of the simulation was to identify possible scenarios determining the senescence of breeders in a population, and not to reproduce the effects of an agespecific decline in reproduction.

We modelled the dynamics of breeders and floaters in the population. The age at first reproduction was set as five years, and the starting breeding population size was set at 15 pairs, which is the number seen in the Doñana population at saturation. The range for floater mortality within settlement areas (i.e. temporary settling zones used during dispersal by non-breeders) was set at $5 \%$ to $40 \%$, reflecting the values we recorded in the field (an annual mortality of 0.2690 .30 was found for unpaired eagles; Ferrer et al. 2004). Increases in floater mortality could be due to environmental stochasticity (e.g. natural catastrophes, habitat destruction and fragmentation) or human-induced deaths (e.g. poisoning and electrocution).

Previous analyses (Penteriani et al. 2005a, 2005b, 2006, 2008) have indicated that environmental stochasticity has a stronger influence on the whole population when variations in environmental conditions occur in a synchronous way (i.e. contemporaneously within settlement and breeding areas), so the simulations were performed under the less adverse scenario (i.e. when variations in environmental conditions occur asynchronously).

All the simulations started at time $\mathrm{t}=0$ with 15 pairs and 15 floaters (to simulate the real situation). We ran 4000 simulations of 100 years each. At the end of each series of 100 simulations, the model generated the mean age of breeders and number of floaters for the 100-year period in each simulation. The output of each simulation contained statistics on the population census for each interaction (1 year). The population dynamics were simulated by individual-based evaluation of three main stochastic functions whose behaviours are believed to be driven by the internal status of the given individual. All numerical values conditioning the shape of the probability distributions were estimated from actual data in the historic series.

The function 'death' computes a Boolean value stating whether the given individual will die during the current year. The value returned by this function is TRUE (the individual will die) if:

$\mathrm{rBA}(\mathrm{a})+(1-\mathrm{A}(\mathrm{a})) \mathrm{B}(\mathrm{s})$

where: $\mathrm{a}$ is the age of the individual; $\mathrm{s}$ is the status (chick, floater, breeder) of the individual; $\mathrm{r}(0 \mathrm{Br} 5)$ is a uniformly distributed random deviate; $\mathrm{A}(\mathrm{a})$ is the continuous probability distribution:

$$
\mathrm{A}(\mathrm{a})=1-1=\left[1+(\mathrm{a}=30)^{10}\right]
$$


describing the probability of death as a function of the individual's age; and $\mathrm{B}(\mathrm{s})$ is the randomised discrete function:

$\mathrm{B}(\mathrm{s})=\mathrm{knP}(\mathrm{s})$

describing the probability of accidental death as a function of the individual's status. Finally, $\mathrm{k}$ and $\mathrm{n}$ are normally distributed random variates with mean 1.0 and standard deviation 1.0, $\mathrm{P}(\mathrm{s})$ is a discrete function returning 0.6 for chicks, 0.06 for breeders and a value in the range $0.05-0.40$ for floaters. The expression $A+(1-A)>B$ implements the logic or operator for the two probability distributions. The value of the random deviate $\mathrm{k}$ is fixed for each year, representing environmental stochasticity. The spatial correlated or uncorrelated effect of stochasticity on breeders and floaters discussed herein was simulated by generating two different values of $\mathrm{k}$, one for breeders and one for floaters (asynchronous stochasticity).

The function 'couple' computes a suitable partner for the individual under consideration. If the individual is a nonbreeder adult, this function generates a list of all potential partners and randomly chooses one of them (if the list is not empty). Each candidate (non-breeder adult of the opposite sex) in the population is tested and included in the list with a density-dependent probability function of the number of couples in the population (see the Appendix in Penteriani et al. 2006 for more details). If a partner is found (i.e. the list is not empty), both the individual being evaluated and the chosen partner are tagged as breeders. The breeders that lost their mates entered the list of potential partners, so with a floater shortage the re-pairing of a breeder that lost its mate is possible because two other breeders that lost their mates can pair up (i.e. they do not need to wait for a floater). As the model is spatially unstructured (i.e. selection of a mate occurs at random from all potential mates within the population), we did not simulate the possibility that selection could act to promote behaviours that might promote the fitness of lineages at the expense of individual fitness (see the last sub-heading of this paper).

The simulation proceeded by iterative evaluation of the 'death' and 'couple' functions each year for each individual in the population, and updated the internal status of each individual as appropriate.

As the real situation on which the simulations were based is a small population at saturation level with a fixed number of breeding sites, we cannot expect that, under a scenario of high numbers of aged floaters, the number of breeding pairs should increase by floaters pairing up. Evidently, if both partners of a pair die in the same year they can be replaced by two floaters.

In addition, (a) the order in which events occur to individuals was determined at random; and (b) the order in which death and breeding were evaluated was randomly chosen, with a $75 \%$ probability of it being 'death-breed' and a $25 \%$ probability of the reverse order. These probabilities were chosen because successful rearing of a chick depends on parental care during $75 \%$ of the year.

Visual debugging of the simulations was performed to prevent errors in the model from generating false outputs (Grimm 2002, Grimm et al. 2006). When simulations were run again, and when the population structure yearby-year was examined during the visual debugging, the following characteristics were observed: (a) once a breeder died, floaters quickly filled the vacant position (in the next year) and the population was always at its saturation level. However, this occurred only for high floater availability (i.e. low floater mortality); (b) an increase in floater mortality caused a corresponding decrease in floater availability, meaning that not all vacancies in the breeding population were filled (i.e. decreased recruitment). This occurred because our model includes a specific probability that determines new pair formation (the function 'couple'). For low numbers of floaters the probability of a new pair forming is very low. In fact, our simulation was not deterministic (e.g. each time a breeder died it was replaced by an adult floater of the same sex), but mating was under a probability constraint and difficulties in pair formation could also appear when the number of floaters was not $n=0$ (see also the function 'couple'); and the decrease in floater numbers and consequent reduced availability of potential mates able to fill vacancies in the breeding territories caused an increase in the age of breeders and, consequently, in the probability of a given breeder's death before the end of the breeding cycle, due to its older age.

\section{Results and discussion}

Within the simulated scenario of a small population of eagles, the mean age of the breeding population showed changes and trends (Fig. 1) depending on: (a) different rates of floater mortality due to the occurrence of environmental stochasticity within settlement areas; (b) the probability of death as a function of the age and status (breeders vs floaters) of individuals; (c) the density-dependent probability of pair formation and stable environmental conditions in the breeding areas; and (d) the density-dependent fecundity and consequent availability of new floaters in the next year.

\section{The floater shortage senescence}

For a population with a low number of floaters (fewer than approximately 40 individuals in the current simulation, Fig. 1), there were relatively few individuals available to fill vacancies within the breeding population due to high mortality rates (i.e. $35-40 \%$ ), and the breeding population was predominantly characterised by old breeders. This scenario, which is a direct consequence of high floater mortality during dispersal, was mirrored by the age increase in the breeding fraction of the population. Because breeders grew old without automatic replacement by floating juveniles, we can call this situation 'floater shortage senescence'.

When the levels of floater mortality were lower (e.g. 15-30\%) and the pool of individuals potentially able to enter as new, younger breeders was consequently bigger, the mean age of breeders decreased and remained generally stable around a given value (i.e. twelve years old in the current simulation; Fig. 1). 


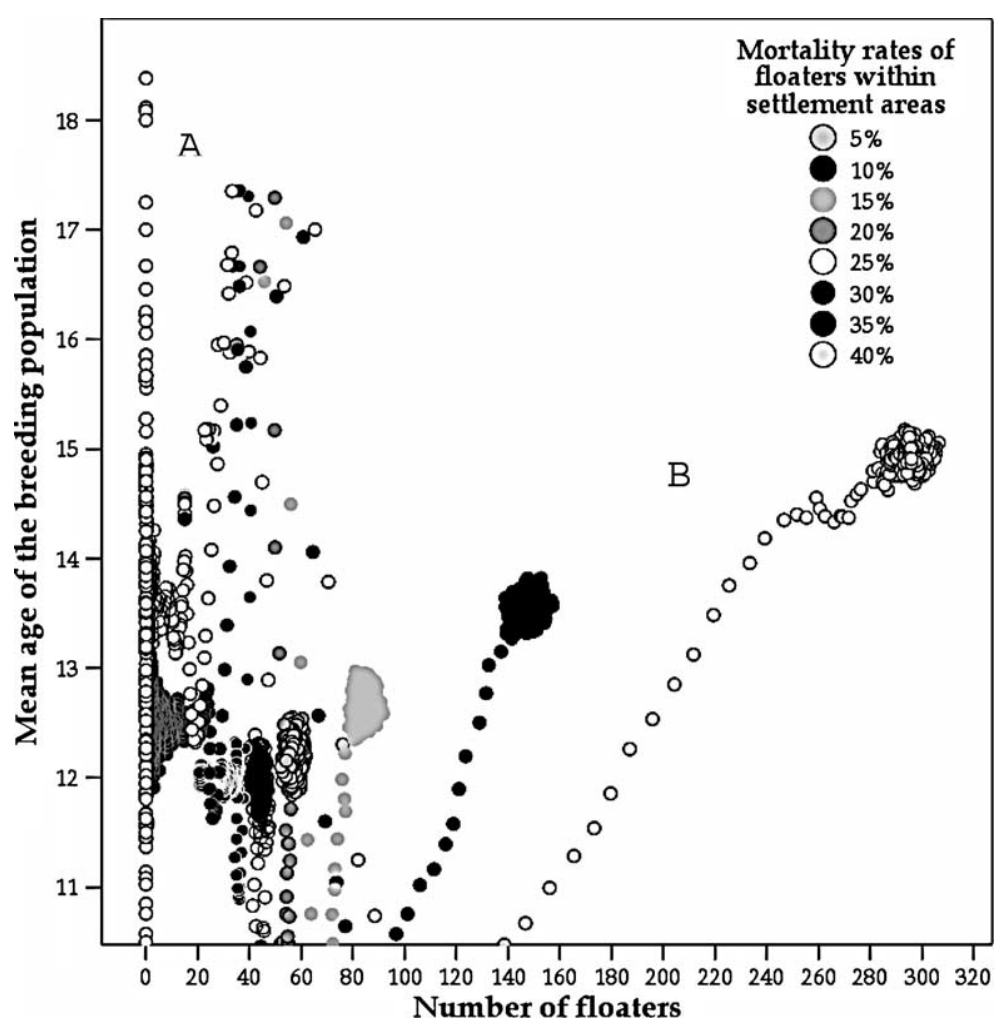

Figure 1. Two different scenarios of individual senescence. Scenario A, which represents 'floater shortage senescence', is due to high mortality rates within settlement areas, and subsequent reduction in the pool of floaters. As a consequence, there are few individuals available to fill breeding vacancies, and the breeding population becomes characterised by older individuals. In scenario B, which represents the so-called 'Florida effect', both the breeding and floating populations senesce because environmental stability allows high breeder survival (determining rare mate replacements and territory vacancies), while low non-breeder mortality allows the formation of an important pool of floaters that senesce due to the saturation of breeding sites.

\section{The Florida effect}

Intriguingly, and probably not so obviously, an increase in the age of the breeding population could also occur in populations with very low floater mortality rates $(5-10 \%$, yielding high numbers of potential new mates wandering close to the breeding sites), and when breeding populations were remarkably free from factors that could cause catastrophic reduction of population numbers (i.e. they were in a stable environment; sensu Mertz 1971). This scenario represents a 'heaven of aged individuals,' where low mortality within the reproductive areas presumably allows territory owners to become older and older, while floaters have a longer lifespan in which to search or queue (sensu Kokko and Sutherland 1998) for a vacant breeding site. As a direct consequence, when a floater enters the breeding population following the death of a previous territory owner, it is already an older breeder due to the long wait for a vacancy (Fig. 2). Under such conditions, new breeders do not refresh the breeding pool, which continues to age, and the breeding areas become characterised by old breeding individuals and old individuals wandering and waiting for a breeding opportunity. This phenomenon, whereby a given area becomes predominantly inhabited by older individuals (both breeders and floaters) because of low mortality rates due to stable conditions, is herein termed the 'Florida effect'. This is because Florida is famous for being largely inhabited by old people; it is called the place "... where old people come to grow older... and older people come to die" (<http://doom.sinosplice.com/2006/02/06/>), and has the largest number of migrating older movers (people 65-84 years of age) in the USA (US Census Bureau 2003).

Do floater shortage senescence and the Florida effect really occur?

The question arises as to whether floater shortage senescence and the Florida effect actually occur among animal (meta)populations. The first scenario is relatively easy to understand, and several real examples have been previously reported. When elevated rates of mortality during dispersal reduce the floater pool, and breeders experience low rates of mortality, and breeding populations may reach advanced ages and thus become subject to senescent deterioration. One of the most famous examples of this occurs in populations of the California condor, Gymnogyps californianus, in which: (a) senescent deterioration may have a tremendous effect on the net reproductive rate (Mertz 1971); and (b) populations cannot remain stable if annual juvenile mortality exceeds 13-15\% (Verner 1978, Meretski et al. 2000). Similarly, Noon and Biles (1990) found the effects of senescence to be most pronounced in a population of spotted owls 


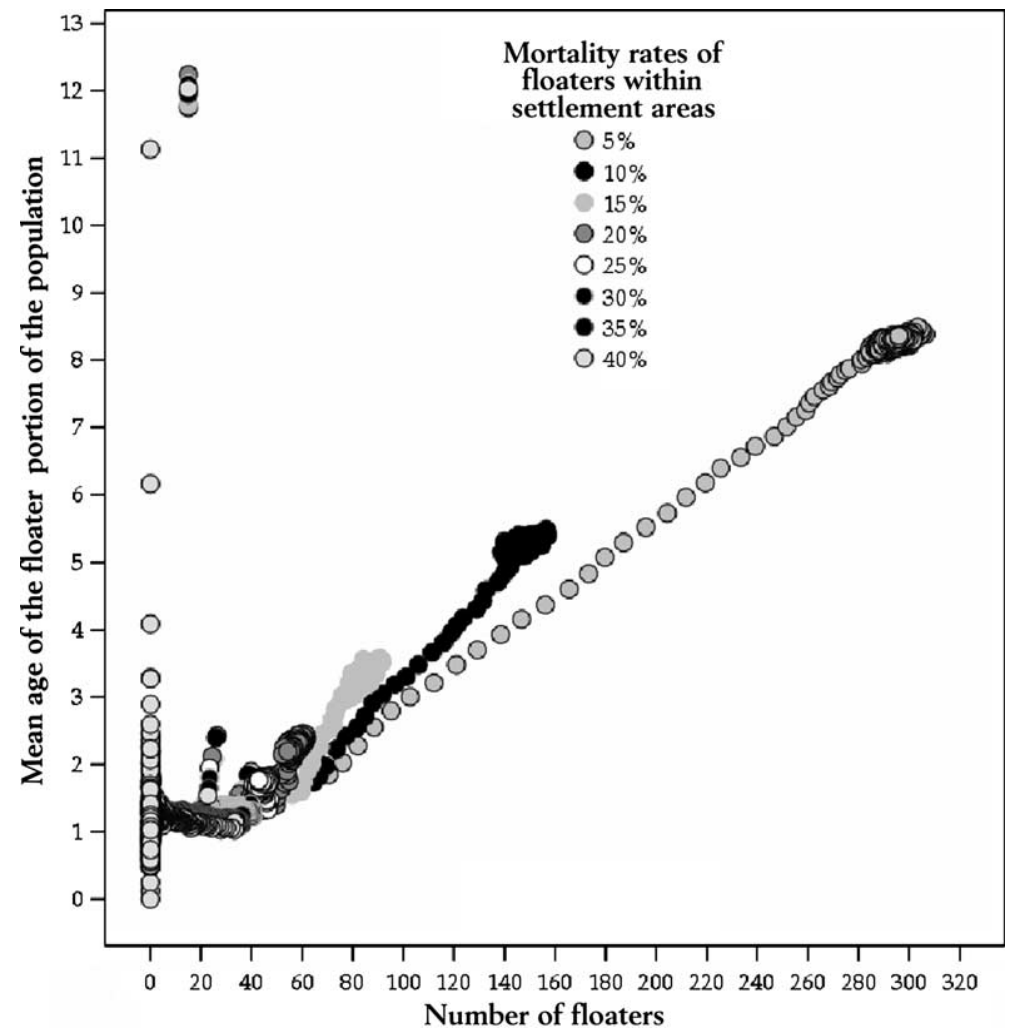

Figure 2. When there is a large pool of floaters (due to low mortality rates in the settlement areas), new breeders may enter the breeding population at a relatively advanced age if the environmental conditions in the breeding areas are stable enough to allow territory owners to live longer.

Strix occidentalis caurina when adult survival rates were high and juvenile survival rates were low.

In contrast, the Florida effect is less intuitive. Firstly, it requires a stable environment (sensu Mertz 1971) in which breeder survival is high, and mate replacement (in the case of long-lived species) or territory vacancies are quite rare events. Secondly, low mortalities in the settlement areas or during dispersal allow formation of an important pool of floaters that senesces along with the breeding population, due to the saturation of breeding sites. The availability of a large pool of old floaters may avoid immediate numerical declines in the breeding population (because each vacancy can be more or less promptly filled by a floater), but will contribute to the overall senescence of the population.

\section{Could intrasexual competition represent an evolutionary response to individual senescence?}

This leads to the question of whether mechanisms have evolved to limit the occurrence of the Florida effect in animal populations. Necessarily, such mechanisms should allow the oldest territory owners or breeding individuals to be evicted by younger individuals. We therefore speculate that some behavioural examples of intraspecific competition (mainly intrasexual competition) could be an evolutionary response to the senescence of individuals in animal populations in stable environments. For example, one of the most common behaviours aimed at limiting individual senescence could be the male-male aggressive encounters, whereby the most forceful individuals inseminate the highest number of females. This generally precludes reproduction of the oldest individuals, as reported by Clutton-Brock (1988), Hinde and Bateson (1991), Møller (1994) and Trivers (2002): (a) the malemale aggressions in harem-forming mammals such as cervids and seals; or (b) the fights among lekking species like grouses. Another possibility is age-dependent male mobility when females are a dispersed resource, whereby more mobile younger males will have access to a larger number of available females. With respect to female choice being an important evolutionary force, the selection of older males may be disadvantaged where females select mates on the basis of attributes such as plumage, display, size of armaments, sexual competence or vigorous courtship (Trivers 2002). Thus, while evolution of female mate preferences may have been directed at male traits that reliably reflect male quality (Møller 1994), such sexual selection may also be an indirect way (or a preliminary step) by which natural selection can reduce individual senescence in animal populations. This hypothesis is consistent with the suggestion that adaptive death plays a crucial role in population dynamics (Travis 2004, Dytham and Travis 2006, Mitteldorf 2006).

Under such a scenario, the populations at highest risk for senescence are those with floaters who wait passively for territory to come available, rather than actively challenging territory owners (Smith 1984, Stutchbury and Robertson 1987, Zack and Stutchbury 1992, Ens et al. 1995, Walls and Kenward 1995, Kokko and Sutherland 1998, Pen and 
Weissing 2000). Thus, when the right moment has come, dying and not to make sex is not necessarily a bad thing.

Acknowledgements - This paper was greatly improved by the helpful comments, opinions and criticisms of Tim Benton, Josh Mitteldorf and Justin Travis.

\section{References}

Abrams, P. A. 1991. The fitness costs of senescence: the evolutionary importance of events in early adult life. - Evol. Ecol. 5: 343-360.

Angelier, F. et al. 2006. Effect of age, breeding experience and senescence on corticosterone and prolactin levels in a longlived seabird: the wandering albatross. - Gen. Comp. Endocrinol. 149: 1-9.

Bonduriansky, R. et al. 2008. Sexual selection, sexual conflict and the evolution of ageing and lifespan. - Funct. Ecol. 22: 443-453.

Bourke, A. F. G. 2007. Kin selection and the evolutionary theory of aging. - Annu. Rev. Ecol. Evol. Syst. 38: 103-128.

Catry, P. et al. 2006. Senescence effects in an extremely long-lived bird: the grey-headed albatross Thalassarche chrysostoma. - Proc. R. Soc. Lond. B 273: 1625-1630.

Clutton-Brock, T. H. 1984. Reproductive effort and terminal investment in iteroparous animals. - Am. Nat. 123: 212.

Clutton-Brock, T. H. 1988. Reproductive success. - Chicago Press.

Curio, E. 1983. Why do younger birds reproduce less well? - Ibis 125: 400-404.

Doncaster, C. P. 2003. Evolution of indefinite generation lengths. - Biol. J. Linn. Soc. 80: 269-280.

Dytham, C. and Travis, J. M. J. 2006. Evolving dispersal and age at death. - Oikos 113: 530-538.

Ens, B. J. et al. 1995. The despotic distribution and deferred maturity: two sides of the same coin. - Am. Nat. 146: 625-650.

Ferrer, M. 2001. The Spanish imperial eagle. - Lynx Editions.

Ferrer, M. and Penteriani, V. 2008. Non-independence of demographic parameters: positive density-dependent fecundity in eagles. - J. Appl. Ecol. 45: 1453-1459.

Ferrer, M. et al. 2004. Density-dependent age of first reproduction as a buffer affecting persistence of small populations. - Ecol. Appl. 14: 616-624.

Grimm, V. 2002. Visual debugging: a way of analyzing, understanding, and communicating bottom-up simulation models in ecology. - Nat. Resour. Modell. 15: 23-38.

Grimm, V. et al. 2006. A standard protocol for describing individual-based and agent based models. - Ecol. Modell. 198: $115-126$.

Hamilton, W. D. 1966. The moulding of senescence by natural selection. - J. Theor. Biol. 12: 12-45.

Hatch, M. I. and Westneat, D. F. 2007. Age-related patterns of reproductive success in house sparrows Passer domesticus. - J. Avian Biol. 38: 603-611.

Hayflick, L. 2000. The future of ageing. - Nature 408: 267-269.

Hinde, R. A. and Bateson, P. P. G. 1991. The development and integration of behaviour. - Cambridge Univ. Press.

Kokko, H. and Sutherland, W. J. 1998. Optimal floating and queuing strategies: consequences for density dependence and habitat loss. - Am. Nat. 152: 354-366.
Le Page, C. and Cury, P. 1995. Age dependent fecundity and the dynamics of a density-dependent population model. - Math. Comput. Modell. 21: 13-26.

Meretski, V. J. et al. 2000. Demography of the California condor: implications for reestablishment. - Conserv. Biol. 14: 957-967.

Mertz, D. B. 1971. The mathematical demography of the California condor population. - Am. Nat. 105: 437-453.

Mitteldorf, J. 2006. Demographic homeostasis and the evolution of senescence. - Evol. Ecol. Res. 8: 1-14.

Monaghan, P. et al. 2008. The evolutionary ecology of senescence. - Funct. Ecol. 22: 371-378.

Møller, A. P. 1994. Sexual selection and the barn swallow. - Oxford Univ. Press.

Newton, I. 1989. Lifetime reproduction in birds. - Academic Press.

Nisbet, I. C. T. 2001. Detecting and measuring senescence in wild birds: experience with long-lived seabirds. - Exp. Gerontol. 36: 833-843.

Noon, B. R. and Biles, C. M. 1990. Mathematical demography of spotted owls in the Pacific Northwest. - J. Wildlife Manage. 54: $18-27$.

Nussey, D. H. et al. 2006. The rate of senescence in maternal performance increases with early-life fecundity in red deer. - Ecol. Lett. 9: 1342-1350.

Partridge, L. and Barton, N. H. 1993. Optimality, mutation and the evolution of ageing. - Nature 362: 305-311.

Pen, I. and Weissing, F. J. 2000. Optimal floating and queuing strategies: the logic of territory choice. - Am. Nat. 155: 512-526.

Penteriani, V. et al. 2005a. Environmental stochasticity in dispersal areas can explain the 'mysterious' disappearance of breeding populations. - Proc. R. Soc. Lond. B 272: 1265-1269.

Penteriani, V. et al. 2005b. Floater survival affects population persistence. The role of prey availability and environmental stochasticity. - Oikos 108: 523-534.

Penteriani, V. et al. 2006. Floater dynamics can explain positive patterns of density-dependence fecundity in animal populations. - Am. Nat. 168: 697-703.

Penteriani, V. et al. 2008. Floater mortality within settlement areas can explain the Allee effect in breeding populations. - Ecol. Modell. 213: 98-104.

Ricklefs, R. E. and Cadena, C. D. 2007. Lifespan is unrelated to investment in reproduction in populations of mammals and birds in captivity. - Ecol. Lett. 10: 867-875.

Roff, D. A. 1992. The evolution of life histories: theory and analysis. - Chapman and Hall.

Ronce, O. et al. 1998. Natal dispersal and senescence. - Proc. Natl Acad. Sci. 95: 600-605.

Sæther, B.-E. 1990. Age-specific variation in reproductive performance of birds. - Curr. Ornithol. 7: 251-283.

Simons, T. R. 1984. A population model of the endangered Hawaiian dark-rumped petrel. - J. Wildlife Manage. 48: 1065-1076.

Smith, S. M. 1984. Flock switching in chickadees: why be a winter floater? - Am. Nat. 123: 81-98.

Stearns, S. C. 1992. The evolution of life histories. - Oxford Univ. Press.

Stutchbury, B. J. and Robertson, R. J. 1987. Behavioral tactics of subadult female floaters in the tree swallow. - Behav. Ecol. Sociobiol. 20: 413-419.

Travis, J. M. J. 2004. The evolution of programmed death in a spatially structured population. - J. Gerontol. 59A: 301-305.

Trivers, R. 2002. Natural selection and social theory. - Oxford Univ. Press. 
US Census Bureau 2003. Internal migration of the older population: 1995 to 2000. - US Dept Commerce Econ. Stat. Adm.

Vaupel, J. W. et al. 2004. The case for negative senescence. - Theor. Popul. Biol. 65: 339-351.

Verner, J. 1978. California Condor: status of the recovery effort. - General Tech. Rep. PSW-28. US For. Service.

Walls, S. S. and Kenward, R. E. 1995. Movements of radio-tagged common buzzards Buteo buteo in their first year. - Ibis 137: 177-182.
Williams, P. D. and Day, T. 2003. Antagonistic pleiotropy, mortality source interactions, and the evolutionary theory of senescence. - Evolution 57: 1478-1488.

Zack, S. and Stutchbury, B. J. 1992. Delayed breeding in avian social systems: the role of territory quality and "floater" tactics. - Behaviour 123: 194-219. 